\title{
Correction to: Radiomics on multi-modalities MR sequences can subtype patients with non-metastatic nasopharyngeal carcinoma (NPC) into distinct survival subgroups
}

\author{
En-Hong Zhuo ${ }^{1} \cdot$ Wei-Jing Zhang ${ }^{2} \cdot$ Hao-Jiang $\mathrm{Li}^{2} \cdot$ Guo-Yi Zhang $^{3} \cdot$ Bing-Zhong Jing $^{2} \cdot$ Jian Zhou $^{2} \cdot$ Chun-Yan Cui $^{2}$. \\ Ming-Yuan Chen ${ }^{2} \cdot$ Ying Sun ${ }^{2} \cdot$ Li-Zhi Liu $^{2} \cdot$ Hong-Min Cai ${ }^{1,4}$
}

Published online: 26 April 2019

(C) European Society of Radiology 2019

\section{Correction to: European Radiology \\ https://doi.org/10.1007/s00330-019-06075-1}

The original version of this article, published on 14 March 2019, unfortunately contained a mistake. The following correction has therefore been made in the original: There was a mistake in Table 1. The corrected table is given below.

The online version of the original article can be found at https://oi.org/ 10.1007/s00330-019-06075-1

Li-Zhi Liu

liulizh@sysucc.org.com

Hong-Min Cai

hmcai@scut.edu.cn

1 School of Computer Science and Engineering, South China University of Technology, Guangzhou 510006, Guangdong Province, People's Republic of China

2 State Key Laboratory of Oncology in South China, Collaborative Innovation Center for Cancer Medicine, Guangdong Key Laboratory of Nasopharyngeal Carcinoma Diagnosis and Therapy, Sun Yat-sen University Cancer Center, No. 651 Dongfeng East Road, Yuexiu District, Guangzhou, Guangdong Province, People's Republic of China

3 Department of Radiation Oncology, Cancer Center, First People's Hospital of Foshan, Foshan 528000, Guangdong Province, People's Republic of China

4 Guangdong Provincial Key Lab of Computational Intelligence and Cyberspace Information, South China University of Technology, Guangzhou, People's Republic of China
Table 1 Demographic and clinical characteristics of patients in training and validation cohorts

\begin{tabular}{|c|c|c|}
\hline Variable & $\begin{array}{l}\text { Training cohort } \\
(N=424) \\
\text { No. }(\%) \text { of patients }\end{array}$ & $\begin{array}{l}\text { Validation cohort } \\
(N=234)\end{array}$ \\
\hline \multicolumn{3}{|l|}{ Age (years) } \\
\hline Mean (SD) & $45.3(11.7)$ & $44.4(10.3)$ \\
\hline Median (IQR) & $45(38-53)$ & $44(37.3-50)$ \\
\hline \multicolumn{3}{|l|}{ Sex } \\
\hline Male & $315(74.3)$ & 167 (71.4) \\
\hline Female & 109 (25.7) & 67 (28.6) \\
\hline \multicolumn{3}{|l|}{ T category } \\
\hline $\mathrm{T} 1$ & 93 (21.9) & $79(33.8)$ \\
\hline $\mathrm{T} 2$ & $59(13.9)$ & $25(10.7)$ \\
\hline $\mathrm{T} 3$ & $162(38.2)$ & 74 (31.6) \\
\hline $\mathrm{T} 4$ & $110(25.9)$ & $56(23.9)$ \\
\hline \multicolumn{3}{|l|}{$\mathrm{N}$ category } \\
\hline N0 & $104(24.5)$ & $54(23.1)$ \\
\hline N1 & $256(60.4)$ & $150(64.1)$ \\
\hline $\mathrm{N} 2$ & $59(13.9)$ & $23(9.8)$ \\
\hline N3 & $5(1.2)$ & $7(3.0)$ \\
\hline \multicolumn{3}{|l|}{ Overall stage } \\
\hline I & $37(8.7)$ & $29(12.4)$ \\
\hline II & $91(21.5)$ & 67 (28.6) \\
\hline III & $182(42.9)$ & $76(32.5)$ \\
\hline IV-a & $114(26.9)$ & $62(26.5)$ \\
\hline \multicolumn{3}{|l|}{ Follow-up time } \\
\hline Median (IQR) & $63.0(56.1-67.1)$ & $62.9(57.1-66.3)$ \\
\hline
\end{tabular}

$S D$ standard deviation, $I Q R$ interquartile range

Publisher's note Springer Nature remains neutral with regard to jurisdictional claims in published maps and institutional affiliations. 